Musées, Patrimoine et Culture scientifiques et techniques

$156 \mid 2014$

novembre-décembre 2014

\title{
Le recensement des Herbiers de France : un nouvel enjeu pour la connaissance
}

\section{Louise Boulangeat}

\section{OpenEdition Journals}

Édition électronique

URL : http://journals.openedition.org/ocim/1457

DOI : $10.4000 /$ ocim. 1457

ISSN : 2108-646X

\section{Éditeur}

OCIM

\section{Édition imprimée}

Date de publication : 1 novembre 2014

Pagination : 12-16

ISSN : 0994-1908

\section{Référence électronique}

Louise Boulangeat, « Le recensement des Herbiers de France : un nouvel enjeu pour la connaissance », La Lettre de I'OCIM [En ligne], 156 | 2014, mis en ligne le 01 novembre 2016, consulté le 01 mai 2019. URL : http://journals.openedition.org/ocim/1457 ; DOI : 10.4000/ocim.1457

Ce document a été généré automatiquement le 1 mai 2019.

Tous droits réservés 


\section{Le recensement des Herbiers de France : un nouvel enjeu pour la connaissance}

Louise Boulangeat

Planche d'herbier de Nymphaea lotus, herbier de Montpellier, université Montpellier 2.

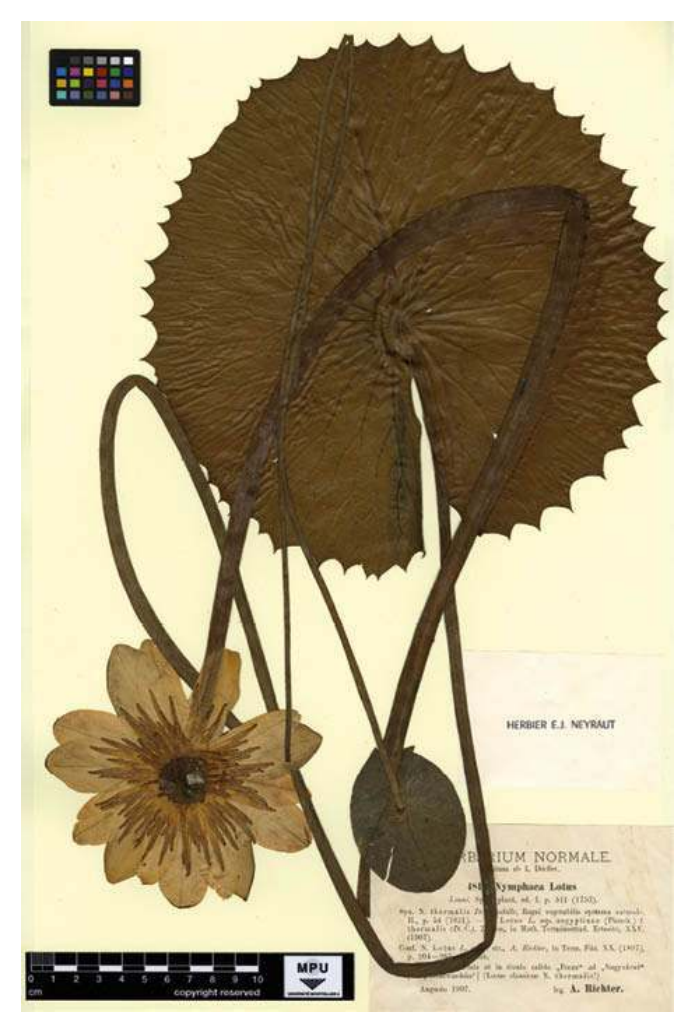

(C) UM2/Sonnet 
1 Dans plusieurs régions de France, des recensements de collections d'herbiers ont eu lieu ces dernières années. Ainsi, entre 2008 et 2011, une enquête a été menée en LanguedocRoussillon afin de dénicher les herbiers de la région. L'étude a répertorié plus de 70 lieux différents : des institutions publiques telles que des universités, musées, archives, mais aussi dans des bibliothèques, collèges, lycées. Ce travail a permis d'informer les propriétaires sur l'importance de ces herbiers, la façon de les conserver, et de répertorier les personnes à contacter pour toutes précisions complémentaires concernant la conservation et la gestion de leurs herbiers.

2 Afin d'étendre cette connaissance des herbiers au niveau national, l'association Tela Botanica a pour mission, dans le cadre du projet ANR eReColNat, de faire le lien entre toutes les études déjà menées et de lancer des dynamiques régionales afin de rassembler sur la plateforme CoEL (Collections En Ligne) toutes les métadonnées sur les herbiers. Pour cela, elle a élaboré une méthodologie et réalisé une fiche d'expertise des herbiers qui permettent de suivre un protocole homogène pour chaque nouvelle étude régionale. Les résultats de ces expertises sont ensuite publiés sur l'outil Internet CoEL. Ils sont aussi envoyés à la base de données internationale de l'Index Herbariorum.

\section{De l'intérêt des herbiers}

3 À partir du XVIe siècle, les facultés de médecine ont constitué des jardins des simples avec des herbiers permettant aux praticiens d'étudier les plantes et de les utiliser dans leurs prescriptions. Au cours des siècles qui ont suivi, les grandes explorations naturalistes ont rassemblé des herbiers volumineux, contenant de nombreuses plantes jusqu'alors inconnues. Ces herbiers historiques sont conservés dans des institutions spécialisées qui en assurent la préservation et en permettent la consultation par les botanistes du monde entier, mais aussi chez des particuliers. Au fil des époques, ces herbiers ont été complétés, échangés, revendus ou dissociés rendant leurs accès parfois difficile. En tout état de cause, ils constituent un formidable livre ouvert sur l'histoire des plantes, leurs migrations, leurs disparitions, dont le contenu est aujourd'hui valorisé dans le cadre de grandes problématiques contemporaines comme l'inventaire de la biodiversité, le suivi des changements climatiques ou la lutte contre les plantes envahissantes. Ces collections sont par ailleurs le support privilégié des chercheurs contemporains dans le domaine de la botanique fondamentale et de la génétique.

4 Les spécimens qui ont servi de base à la description des espèces représentent la part la plus précieuse des herbiers, elles constituent la référence en matière de nomenclature botanique. La recherche et la numérisation de ces spécimens, dénommés types nomenclaturaux, a fait l'objet de projets internationaux très importants ces dernières années, notamment avec le programme Global Plants Initiative financé par la Fondation américaine Andrew Mellon qui a permis de numériser et diffuser plus de 2 millions de ces échantillons types. Les progrès récents en matière de séquençage de l'ADN d'échantillons anciens ouvrent une porte sur un contenu jusqu'alors ignoré des herbiers : celui de leur contenu génétique riche de nombreuses perspectives. Toutes ces applications montrent la nécessité qu'il y a à faire le bilan de ces collections, de leur état de conservation, d'inventorier leur contenu et de le rendre disponible aux chercheurs de plus en plus nombreux qui s'intéressent à la biodiversité végétale sous tous ses aspects. Enfin, les herbiers représentent également une source de données historiques intéressante. En 
effet, ils sont accompagnés de carnets de récoltes, d'étiquettes et d'ouvrages, parfois de peintures ou de gravures qui retracent la vie des grands explorateurs et nous informent sur leurs voyages.

\section{La diversité des herbiers et de leurs vocations}

5 Dans la pratique, il existe trois grands types d'herbiers: les herbiers de travail, les herbiers pédagogiques et les herbiers historiques.

6 Les herbiers de travail sont utilisés par les botanistes pour observer et ré-observer un taxon tout au long de l'année. Ils doivent pouvoir être disséqués, observés, mesurés et être disponibles facilement aux chercheurs. Ces herbiers servent principalement à des recherches en systématique et en biogéographie.

7 Les herbiers pédagogiques sont en général très bien disposés, attachés et souvent plastifiés. Il y manque quelquefois des informations de dates et lieux précis de récoltes mais ils peuvent contenir des informations supplémentaires concernant les caractéristiques des plantes et de leur milieu.

Les étagères d'un des six étages de l'herbier de Montpellier, université Montpellier 2.

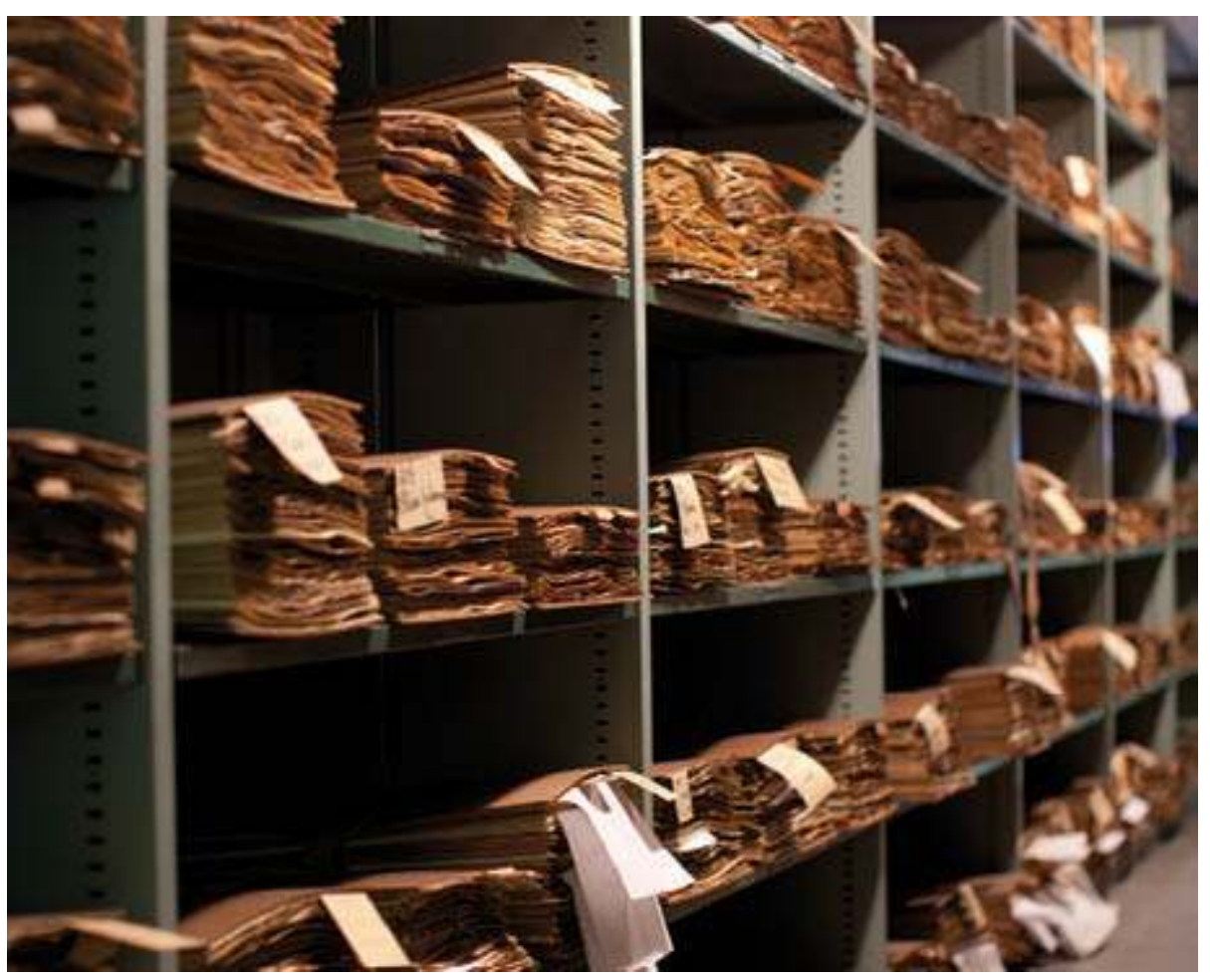

(c) UM2/Sonnet

8 Les herbiers historiques sont des herbiers anciens, collectés par des botanistes dont ils retracent la carrière et les voyages au travers des plantes récoltées.

9 Ces différents herbiers sont conservés chez des propriétaires très divers (institutions, individus) et la taille des collections est très variable, de quelques centaines à quelques centaines de milliers d'échantillons. Selon les régions, ces collections peuvent se répartir de façon très différentes, en très grande majorité dans des institutions (Provence-Alpes- 
Côte d'Azur, Languedoc-Roussillon), mais aussi chez des particuliers (jusqu'à $20 \%$ en région Rhône-Alpes).

Ces herbiers, outils pour le botaniste, font aussi partie du patrimoine (les meubles, les contenants, les papiers). L'enquête permet de retrouver les collections disparues et les informations sur les botanistes dans les correspondances, dans leur manière d'échanger les uns avec les autres, dans leurs activités. Comme le rappelle Andrine Faure, responsable du recensement des herbiers en Rhône-Alpes puis en Languedoc-Roussillon

"l'expertise c'est le plaisir de l'enquête et de découvrir qui était l'auteur de l'herbier, ce qu'il faisait et son caractère. L'herbier est une source incroyable d'informations: on y retrouve des informations sur les relations avec les autres botanistes, des petits mots du type journal intime, très méticuleux ou encore au brouillon...".

11 Mieux connaître une collection va aussi pouvoir mener à la possibilité de mettre en place des expositions, conférences, visites, formations...

\section{Un projet d'envergure nationale}

La France est un des pays les plus riches en terme de collections botaniques, ainsi notre responsabilité collective est-elle engagée sur la façon dont nous gérons nos collections et dont nous portons à connaissance du monde l'ensemble de leurs contenus. Le projet eReColNat financé par l'Agence Nationale pour la Recherche (ANR) et piloté par le Muséum national d'Histoire naturelle qui a débuté en 2013, vise à relever ce défi. L'objectif général est de valoriser 350 ans de collections naturelles sur une plateforme numérique. Ce projet se divise en plusieurs volets : numériser un très large échantillon de planches d'herbiers, numériser les échantillons de référence en paléontologie et zoologie et accompagner le recensement des herbiers publics et privés à l'échelle nationale. Ce dernier volet a pour but de faire un état des lieux des collections botaniques région par région et à les caractériser pour mieux les préserver. Ce travail fait suite aux travaux déjà réalisés en Rhône-Alpes, Languedoc-Roussillon, Provence-Alpes-Côte d'Azur et en Auvergne depuis plusieurs années et prend appui sur les outils développés par Tela Botanica en partenariat avec l'université des Sciences (UM2) de Montpellier.

Le programme eReColNat a été mis en place pour une durée de 5 ans de manière à constituer une plateforme globale de données sur les collections naturalistes françaises (zoologie, paléontologie, herbiers). Cette plateforme permettra aux chercheurs d'avoir un accès facilité à ces collections, ce sera aussi un lieu d'échanges privilégié entre scientifiques. 
Carte de France des collections recensées, disponible en ligne : www.tela-botanica.org/widget:coel:carto.widget:coel:carto

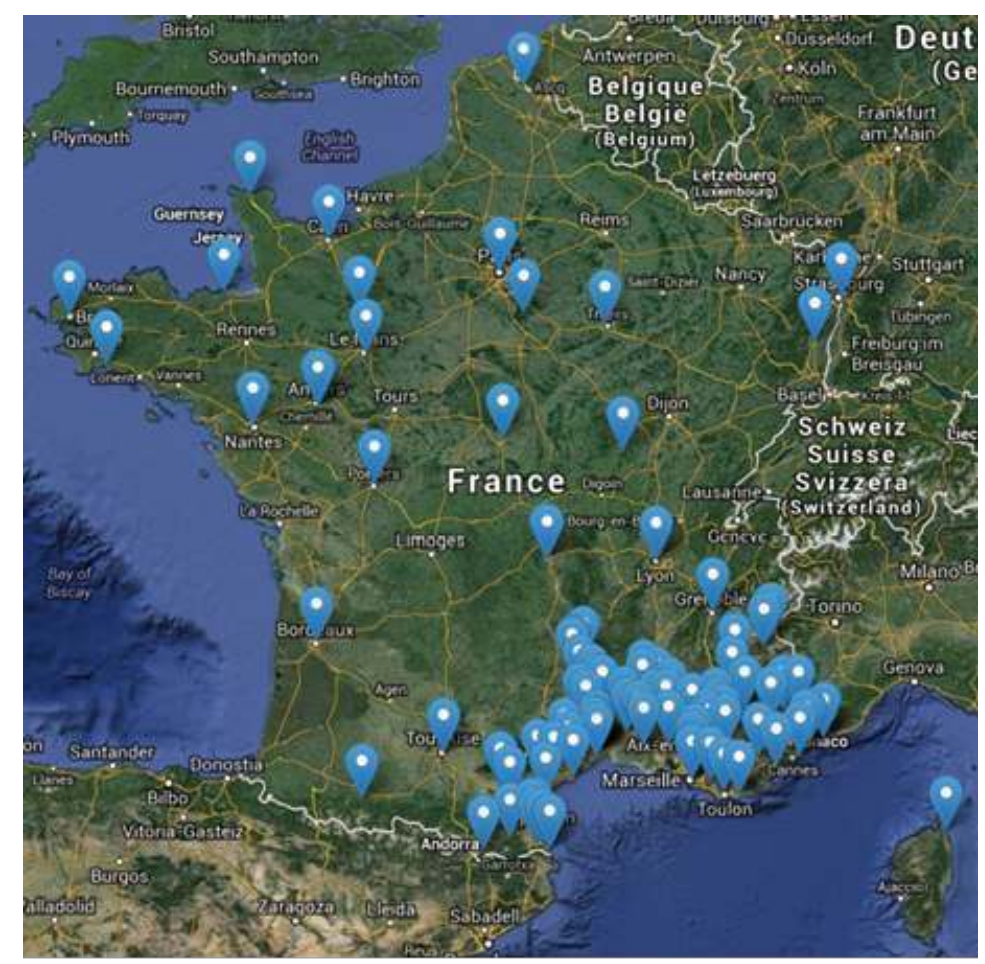

Elle sera ouverte au grand public avec une possibilité de visite virtuelle des collections permettant de consulter et d'imprimer les images numérisées, par exemple pour préparer des animations, des ateliers et des expositions à thème.

D'autre part, le public est d'ores et déjà invité à participer à la documentarisation des images d'herbiers (saisie en base de données des informations manuscrites portées par les planches) dans le cadre d'un programme de sciences participatives nommé "LesHerbonautes" (www.lesherbonautes.mnhn.fr).

16 Au delà du projet eReColNat présenté ci-dessus, le recensement des herbiers français intéresse aussi une communauté plus étendue. C'est pourquoi les données sont mises en valeur sur le site du Global Biodiversity Information Facility.

Enfin, au fur et à mesure de l'avancée du projet, quelques herbiers "hors-France" (notamment en Afrique du Nord et de l'Ouest) participent au recensement. C'est pourquoi nous travaillons étroitement avec l'Index Herbariorum. Cet Index géré par le New York Botanical Garden, référence principalement les plus grands herbiers du monde mais tend vers un recensement exhaustif des herbiers publics mondiaux.

\section{Une méthodologie adaptée}

18 À partir de l'expérience tirée du travail de recensement des herbiers en Rhône-Alpes (2006), Auvergne (2010), Provence-Alpes-Côte d'Azur (2011) et Languedoc-Roussillon (2011), nous avons élaboré une méthodologie permettant d'étendre le projet à l'échelle nationale de façon cohérente. Cette méthodologie a été conçue de manière à répondre 
aux objectifs de recenser le plus exhaustivement possible les herbiers publics et privés de France dans les délais imposés par le programme eReColNat.

Dans un premier temps, nous réunissons un comité de pilotage régional constitué par des botanistes et des conservateurs de musées concernés localement par la mise en place d'une dynamique autour des herbiers. Ce comité de pilotage veille notamment au bon déroulement du recensement régional, en lien avec le programme national. Ce comité de pilotage, accompagné par Tela Botanica, est aussi en charge d'une éventuelle recherche de financements permettant de recruter la personne qui sera en charge du recensement régional.

Dans un deuxième temps, une enquête est lancée de façon très large afin de repérer un maximum de collections (courriers aux institutions, messages dans la presse, contacts avec les sociétés botaniques, les musées, les archives, les bibliothèques, les lycées agricoles...). Nous avons noté que les moyens les plus efficaces étaient de diffuser l'information lors de sorties botaniques locales ou d'événements régionaux. En effet, les herbiers étant parfois tombés dans l'oubli, c'est par le bouche à oreille que nous pouvons le plus facilement retrouver les collections oubliées.

Lorsque les herbiers sont localisés, le comité de pilotage ou son mandataire va visiter chacune des collections afin de les expertiser. Pour cela, il remplit la fiche d'expertise nationale, standardisée selon un standard international (DarwinCore). Cette fiche d'expertise a été élaborée à partir des travaux de recensement des herbiers d'Andrine Faure entre 2006 et 2011. Elle permet de renseigner les métadonnées des collections de manière très précise : taille, état, spécialités, localités, collecteurs...

L'étape suivante consiste à saisir les résultats dans la base de données en ligne CoEL développée spécifiquement pour ce programme. Cette base de données, consultable librement en ligne (www.tela-botanica.org/herbiers) permet aux utilisateurs de contacter directement les propriétaires ou les gestionnaires des collections pour avoir des informations complémentaires ou travailler sur les collections.

Enfin, de manière à valoriser le travail accompli et rendre compte de cette étude, notamment auprès des financeurs, il est important de publier un rapport d'étude, disponible en ligne également.

\section{CoEL, un outil incontournable pour décrire et consulter les collections}

CoEL (Collections En Ligne) est la base de données utilisée pour gérer et consulter les métadonnées des collections d'herbiers. Elle est disponible dans la partie "Botaniqueherbiers" du site de Tela Botanica (contact : coel@tela-botanica.org). Cette base de données libre est ouverte à tous, elle suit les standards internationaux du DarwinCore, les résultats sont ensuite envoyés au Global Biodiversity Information Facility pour être partagés au niveau international. Actuellement, 226 fiches décrivant des institutions détentrices d'herbiers et 1575 fiches décrivant les collections ont été saisies sur cette base de données. Cette large diffusion de l'information permet aux gestionnaires de valoriser leurs collections à l'échelle internationale. 
Un droguier de l'herbier de Montpellier, université Montpellier 2.

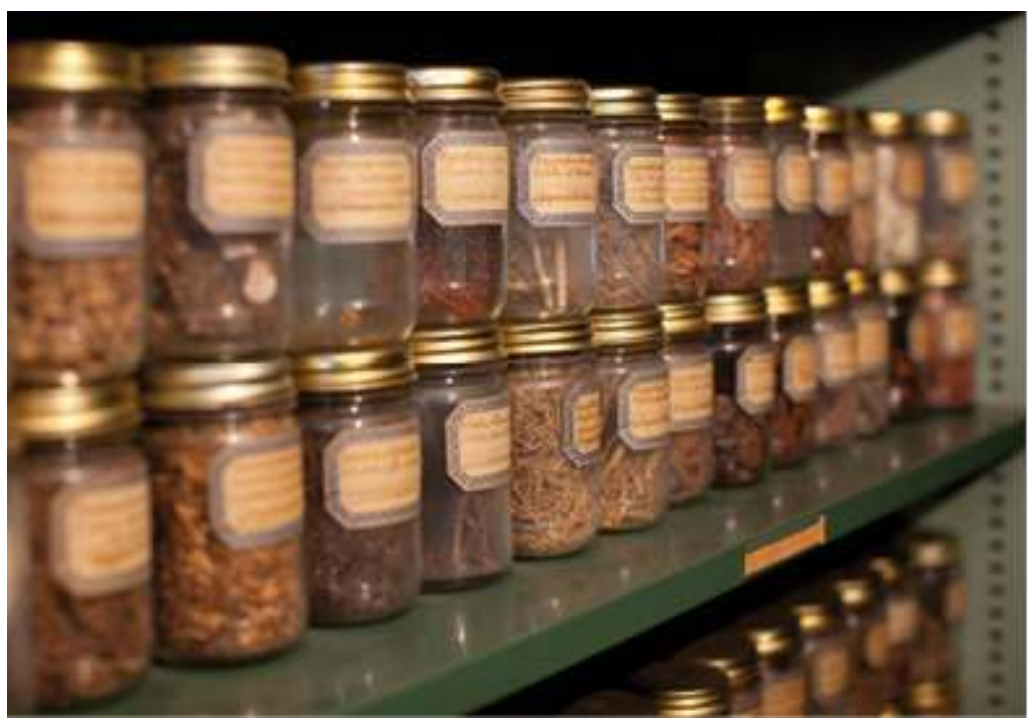

(C) UM2/Sonnet collections contenues dans la base de données selon différents critères. Plusieurs onglets permettent d'accéder aux informations sur les particularités et le contenu des collections (dates, localités...), les botanistes en lien et leurs publications. Une carte interactive est aussi disponible afin de visualiser où visiter et consulter les collections.

Le mode saisie est réservé aux personnes inscrites à Tela Botanica. Il permet d'enregistrer l'intégralité des données rassemblées sur les fiches d'expertise. C'est le mode d'accès privilégié des responsables de recensement régionaux.

2001 : Journée nationale de réflexion sur les herbiers (Montpellier) 2001 : Ouverture du forum "Herbiers" de Tela Botanica

2002 : Colloque Les Herbiers, un outil d'avenir. Tradition et modernité (Lyon)

2003 : Ouverture de la base de données "Herbiers" sur Tela Botanica

2003 : Publication du recensement des herbiers de Franche-Comté

2006 : Publication du recensement des herbiers de Rhône-Alpes

2008-2011 : Recensement des herbiers de Provence-Alpes-Côte d'Azur et Languedoc-Roussillon

2010 : Publication du recensement des herbiers d'Auvergne

2012: Colloque Herbiers publics et privés de Provence-Alpes-Côte d'Azur (Digne-les-Bains)

2013 : Colloque Herbiers, trésors vivants (Bordeaux)

2013 : Début du programme national "Recensement des herbiers de France", dans le cadre de l'ANR eReColNat

\begin{tabular}{|c|c|c|c|}
\hline Région & $\begin{array}{l}\text { Nbre d'adresses } \\
\text { (Dont particuliers) }\end{array}$ & Nbre de collections & Nbre de spécimens \\
\hline Languedoc-roussillon (1) & $86(27)$ & 591 & 4000000 \\
\hline Provence-Alpes-Côte-d'Azur (2) & $51(13)$ & 360 & 1958000 \\
\hline Auvergne ${ }^{(3)}$ & 8 & 121 & 629097 \\
\hline Aquitaine et Poitou-Charente ${ }^{(4)}$ & 165 & 323 & Min. 500000 \\
\hline Franche-Comté ${ }^{(5)}$ & 13 & 100 & 536636 \\
\hline
\end{tabular}




\section{Une étude publique pour des résultats partagés}

Planche d'herbier de Lagurus ovatus, herbier de Montpellier, université Montpellier 2.

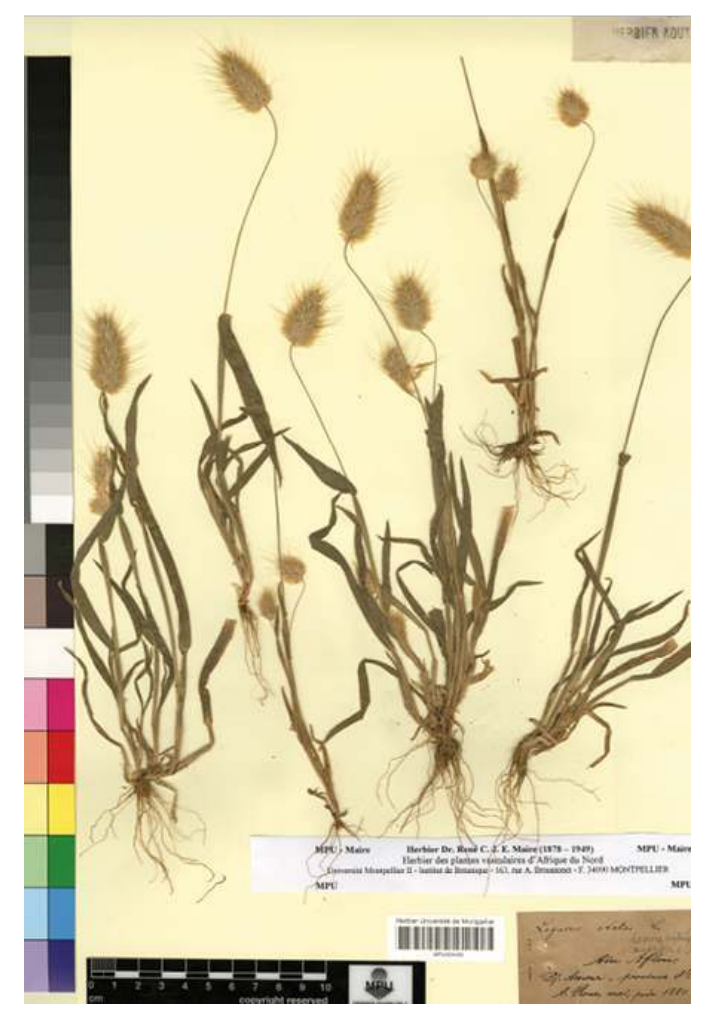

(c) UM2/Sonnet

Le protocole national établi a pour but de caractériser au mieux la collection. Les informations relevées concernent l'institution où est hébergée la collection et ses caractéristiques de conservation, comment contacter la structure et où trouver l'herbier. De même, cette plateforme réunit des informations propres à l'herbier: l'origine géographique, l'époque, le contenu taxonomique, le nombre de spécimens, l'état de conservation... Enfin, cette base de données répertorie aussi les botanistes et les publications liées à la collection.

27 Afin que ces travaux soient efficacement partagés, les données d'expertise sont publiées en ligne et chacun peut suivre les nouvelles contributions via les flux RSS. Cette disposition permet des retours rapides d'informations de la part des visiteurs. Nous avons fait le choix de mettre ces données en licence libre afin qu'elles puissent être réutilisables par tous, à condition de citer les auteurs.

La mise en place de ce projet de recensement national est maintenant bien établie avec les études présentées ci-dessus qui ont eu lieu dans quelques régions mais les investigations se poursuivent partout en France afin de trouver les herbiers non répertoriés et/ou non expertisés. Ainsi, les herbiers seront mieux identifiés ce qui permettra de mieux les protéger, les valoriser et les rendre accessibles à la communauté des botanistes. 


\section{BIBLIOGRAPHIE}

Cazin, A. et Debout, G. Naturalia, Panorama des collections bas-normandes d'histoire naturelle. Centre Régional de Culture Ethnologique et Technique de Basse-Normandie, 2013, 112 p.

Corradini, P. Inventaire des herbiers de Franche-Comté. Les herbiers franc-comtois : un patrimoine insoupçonné, un outil d'avenir. 2003, 81 p.

Durand, M. Inventaire des herbiers publics et privés de la région Provence Alpes Côte d'Azur. Aixen-Provence : Muséum d'Aix-en-Provence, 2011, 75 p.

Faure, A. Inventaire des herbiers publics et privés de la région Languedoc-Roussillon.

Montpellier : Tela Botanica, 2011, $65 \mathrm{p}$.

Lerond, M., Van Haluwyn, C., Asta, J. et Lamy, D. Inventaire des herbiers français de lichens.

Cryptogamie, Bryol. Lichénol, nº 8 (1), 1987, pp. 1-25.

Lassimonne, S.-E. et Lauby, A. Catalogue des collections botaniques du massif central. Moulins :

Auclaire, 1905, 216 p.

Thebaud, G., Delcoigne, A. et Roux, C. Recensement des herbiers d'Auvergne. Clermont-Ferrand : PRES-Clermont, 2010, $40 \mathrm{p}$.

Association Tela Botanica : les outils, l'espace projet et les documents du programme de recensement des herbiers publics et privés de France, www.tela-botanica.org/herbiers

ANR eReColNat : description du programme général, http://recolnat.org

\section{RÉSUMÉS}

Mieux connaître les collections publiques et privées d'herbier à l'échelle nationale et rendre ces informations disponibles, telle est la mission qui a été assignée - dans le cadre du projet ANR eReColNat - à l'association Tela Botanica : cette contribution rappelle la méthodologie suivie pour ce recensement, les objectifs poursuivis et souligne l'intérêt de la démarche

\section{INDEX}

Mots-clés : herbier, recensement

\section{AUTEUR}

\section{LOUISE BOULANGEAT}

Responsable du projet de recensement des herbiers publics et privés de France à Tela Botanica louise@telabotanica.org 